\title{
Análise dos sintomas osteomusculares de professores do ensino fundamental em Matinhos (PR)
}

\author{
Analysis of musculoskeletal symptoms of elementary \\ school teachers in Matinhos (PR)
}

\section{Maria Silvia Martins Mango ${ }^{[a]}$, Mônica Kopplin Carilho ${ }^{[b]}$, Bianca Drabovski ${ }^{[c]}$, Emerson Joucoski ${ }^{[\mathrm{d}]}$, Maurício Camargo Garcia ${ }^{[\mathrm{e}]}$, Anna Raquel Silveira Gomes ${ }^{[\mathrm{f}]}$}

[a] Graduada em Fisioterapia pela Universidade Federal do Paraná (UFPR), Matinhos, PR - Brasil, e-mail: sil_mango@yahoo.com.br

[b] Graduada em Fisioterapia pela Universidade Federal do Paraná (UFPR), Matinhos, PR - Brasil, e-mail: monicarrilho@gmail.com

[c] Mestre em Fisiologia da Performance pela Universidade Federal do Paraná (UFPR), Curitiba, PR - Brasil, e-mail: bibia06@gmail.com

[d] Mestre em Física pela Universidade Federal do Paraná (UFPR), docente do curso de Fisioterapia na Universidade Federal do Paraná (UFPR), Matinhos, PR - Brasil, e-mail: joucoski@gmail.com

[e] Doutor em Zoologia pela Universidade Federal do Paraná (UFPR), docente dos cursos de Oceanografia e Aquicultura da Universidade Federal do Paraná (UFPR), Pontal do Paraná, PR - Brasil, e-mail: mauricio.camargo@ufpr.br

[f] Doutora em Ciências Fisiológicas pela Universidade Federal de São Carlos (UFSCar), docente do curso de Fisioterapia da Universidade Federal do Paraná (UFPR), Matinhos, PR - Brasil, e-mail: annaraquelsg@gmail.com

\section{Resumo}

Introdução: Com a finalidade de investigar a etiologia dos sintomas osteomusculares em professores, estudos tem abordado a relação entre o trabalho docente e as possíveis particularidades da profissão que podem levar ao adoecimento físico dos professores. Objetivo: Analisar os sintomas osteomusculares de professores do ensino fundamental da cidade de Matinhos (PR). Materiais e métodos: Foram avaliados 126 professores (120 mulheres e seis homens, 38,6 \pm 9 anos) por meio de um questionário contendo dados gerais e ocupacionais e pelo questionário nórdico. Análises estatísticas univariadas desses questionários foram realizadas com o teste qui-quadrado. Um modelo linear logístico múltiplo foi estabelecido para predizer as variáveis respostas do questionário nórdico a partir das variáveis independentes do questionário de dados gerais e ocupacionais. Resultados: A presença de dor não indicou associação 
significativa com a idade, realização de atividade física, número de alunos por classe e tempo de profissão $(\mathrm{p}>0,05)$, relacionou-se apenas com o número de classes que lecionavam $(\mathrm{p}=0,02)$. Porém, a presença de dor nos últimos 12 meses teve relação com o impedimento de realizar atividade nos últimos 12 meses e a procura por algum profissional da saúde ( $p>0,05)$. Dos participantes, 95,2\% apresentaram algum sintoma osteomuscular. As áreas mais atingidas foram lombar (51,5\%), região dorsal $(49,2 \%)$, ombro $(49,2 \%)$ e pescoço $(47,6 \%)$. Conclusões: Os professores apresentaram elevada prevalência de sintomas osteomusculares, especialmente na lombar, nos ombros e na região dorsal, os quais, por sua vez, provocaram o impedimento de realizar atividades cotidianas.

Palavras-chave: Doenças musculoesqueléticas. Doenças profissionais. Saúde do trabalhador. Docentes.

\section{Abstract}

Introduction: To investigate the etiology of musculoskeletal symptoms in teachers, studies have addressed the relationship between teaching and possible peculiarities of the profession that can lead to physical illness of teachers. Objective: To identify the occurrence of musculoskeletal symptoms of elementary school teachers in Matinhos (PR). Materials and methods: It was evaluated 126 teachers (120 women and 6 six men, $38.6 \pm 9$ years old) through a questionnaire containing an inventory for general and occupational data and the Nordic questionnaire. In the statistical analysis we compared independent variables from inventory for general and occupational data dependent with dependent variables of Nordic questionnaire by multivariate logistic regression analysis and chi-squared test to compare $(p \leq 0.05)$. Results: The presence of pain did not indicate relationship with age, physical activity, number of students per class or time of profession ( $p>0.05)$, although the number of classes per teacher was significant $(p=0.02)$. However, the pain in the last 12 months was related to prevent activities in the last 12 months and also to assistance by health professional ( $p>0.05)$. Musculoskeletal symptoms occurred in $95.2 \%$ of participants. The most affected regions were: lower back (51.5\%), chest (49.2\%), shoulders (49.2\%) and neck (47.6\%). Conclusion: Thus, teachers presented high prevalence of musculoskeletal symptoms especially in lower back, chest and shoulders, and these prevented daily activities.

Keywords: Musculoskeletal diseases. Occupational diseases. Occupational health. Faculty.

\section{Introdução}

Os distúrbios do sistema musculoesquelético têm despertado a atenção de pesquisadores preocupados com questões relativas à saúde e ao trabalho, em virtude do custo e do impacto na qualidade de vida. Nos anos 80 , os sintomas osteomusculares começaram a se destacar no Brasil, tornando-se um grande problema de saúde pública (1) e adquirindo expressão em número e relevância social $(2,3)$.

É clara a importância do trabalho na vida do homem, pois, além de ser sua fonte de renda, trata-se de onde ele pode se sentir útil, produtivo e valorizado, tendo sua autoestima elevada, contando com a possibilidade concreta de autorrealização. Entretanto, quando realizado com condições inadequadas, o trabalho pode ser nocivo, prejudicando a saúde, provocando doenças, levando à inatividade, encurtando a vida e até causando a morte (4).
As Lesões por Esforços Repetitivos ou Doenças Osteomusculares Relacionadas ao Trabalho (LER/ DORT) representam um dos grupos de doenças ocupacionais mais polêmicos no Brasil e em outros países, e vem assumindo um caráter epidêmico (2). As estatísticas do Instituto Nacional de Seguridade Social (INSS) indicam aumento na concessão de benefícios por DORT (5). Esse quadro patológico é a segunda maior causa de afastamentos laborais (6).

Os afastamentos dos docentes de suas atividades podem estar relacionados com as condições de trabalho a que estão expostos (muito tempo em pé, ou em ambientes não ergonômicos) (7). As atividades repetitivas foram identificadas por Porto et al. (8) como o principal risco da atuação dos docentes, numa análise feita de atendimentos a professores pelo Centro de Estudos de Saúde do Trabalhador (CESAT), durante o período de 1991 a 2001. 
As LER/DORT são danos decorrentes da utilização excessiva, imposta ao sistema osteomuscular, e da falta de tempo para recuperação. Em geral, são caracterizadas pela ocorrência de vários sintomas, concomitantes ou não, de aparecimento insidioso, predominantemente nos membros superiores (2). Embora a causa das DORT seja uma questão não completamente elucidada, prevalece um consenso quanto à natureza multifatorial. Estudos epidemiológicos têm mostrado a relação entre fatores de risco físicos como repetitividade, força muscular e posturas inadequadas, extremas e estáticas, e distúrbios osteomusculares (9). Outros estudos identificam associação entre as DORT e fatores de risco organizacionais e psicossociais, como períodos prolongados de trabalho, altas demandas de trabalho, pressão por tempo e ambiente social de trabalho (9).

Em relação aos professores, as lesões e/ou alterações osteomusculares mais comuns são a protrusão da cabeça e dos ombros, a hiperlordose cervical e lombar, a cervicobraquialgia, a lombociatalgia, as algias vertebrais, a bursite do ombro, a escoliose, as tendinopatias do punho e as síndromes compressivas do complexo punho-antebraço (10). Os sinais e os sintomas referidos englobam dores, parestesias, limitações funcionais, inflamação, diminuição da força muscular (principalmente no tronco e nos membros inferiores), tensão muscular (principalmente no pescoço e na cintura escapular), retrações musculares e limitações articulares, cãibras, cefaleia, problemas circulatórios, irritabilidade geral e estresse orgânico e mental (10), além de sensação de peso e fadiga (2).

Ensinar é uma atividade em geral altamente estressante, com repercussões evidentes na saúde física, mental e no desempenho profissional dos professores. 0 estresse ocupacional pode ser constatado entre os docentes por seus sintomas e pela redução na frequência ao trabalho. Fatores psicológicos ligados ao estresse docente incluem ansiedade, depressão, irritabilidade, hostilidade e exaustão emocional (11).

Alguns estudos têm relatado alta prevalência de sintomas osteomusculares em professores do ensino fundamental em diferentes regiões do Brasil, como Rio Grande do Norte e Bahia $(12,13)$. 0 problema se repete pelo país e faz com que as doenças de quem leciona tornem enfermo o sistema de ensino, podendo ocasionar prejuízos para o aprendizado.

Dessa forma, percebe-se a necessidade de analisar os sintomas osteomusculares dos professores, permitindo a identificação dos problemas, com a participação efetiva dos sujeitos do processo de trabalho, que deve ser replanejado. Trabalho prazeroso é aquele em que cabe ao trabalhador uma parte importante da concepção. Portanto, a inventividade, a criatividade, a capacidade de solucionar problemas, 0 emprego da inteligência é o que deve ser buscado (14).

Por isso, este estudo teve como objetivo avaliar os sintomas osteomusculares relacionados à profissão de professor da rede municipal de ensino de Matinhos (PR).

\section{Materiais e métodos}

A amostra foi composta por professores das escolas municipais de Matinhos, os quais estavam presentes na semana pedagógica na UFPR Setor Litoral, no mês de fevereiro de 2008. Os participantes tinham idade média de 38,6 \pm 9,4 anos, sendo $95,2 \%$ do gênero feminino, e 4,7\%, masculino. Como critérios de inclusão, foram considerados todos os professores efetivos e que estavam trabalhando em salas de aula, diretamente com os alunos, e que estavam presentes no dia da entrega do questionário na semana pedagógica. Foram excluídos os questionários que continham dados insuficientes para a pesquisa.

Como instrumento de avaliação, utilizou-se um questionário autoaplicável com duas partes: 1. Dados gerais e ocupacionais - Abordando questões como idade, estado marital, filhos, realização de atividade física, número de classes que lecionava, número de alunos por sala de aula, tempo de atuação na profissão e rendimento médio pessoal (1); 2. Questionário Nórdico - Para avaliar os sintomas musculoesqueléticos, foi utilizada a parte geral do questionário nórdico, adaptado culturalmente para a língua portuguesa por Barros e Alexandre (15). É composto por uma figura humana dividida em nove regiões anatômicas, na qual o respondente deve relatar a ocorrência dos sintomas considerando os 12 meses e os sete dias precedentes à entrevista, bem como informar a ocorrência de afastamento das atividades rotineiras no último ano (16).

Utilizando tabelas de frequência para as variáveis categóricas e estatísticas descritivas (média e desvio padrão) para as variáveis numéricas, realizou-se uma análise descritiva dos dados. As relações entre as variáveis categóricas foram observadas pelos testes de associação qui-quadrado. Foi aplicado para cada região do corpo abordada pelo questionário, relacionando: impedimento de realizar atividade nos últimos 12 meses com a presença de dor nos últimos 
12 meses, a consulta a algum profissional da saúde nos últimos 12 meses com a presença de dor nos últimos 12 meses, e a consulta a algum profissional da saúde nos últimos 12 meses com problemas nos últimos sete dias. Análises estatísticas regressivas foram aplicadas utilizando-se as variáveis explicadoras (independentes) do questionário de dados gerais e ocupacionais, e as variáveis respostas (dependentes) do questionário nórdico por meio de um modelo linear logístico multivariado ( $\mathrm{p} \leq 0,05)$.

0 estudo foi aprovado pelo Comitê de ética no Setor de Ciências da Saúde da Universidade Federal do Paraná - Curitiba, sob registro CEP/ SD: 0079.0.091.000-08. Todos os sujeitos assinaram um termo de consentimento livre e esclarecido (Resolução n. 196/96 do Conselho Nacional de Saúde) de acordo com as normas do Comitê de Ética da Instituição, conforme a declaração de Helsinki.

\section{Resultados}

Cento e vinte e nove professores responderam os questionários, porém três foram excluídos por insuficiência de dados, tornando a amostra composta por 126 indivíduos. Dos participantes, 40,4\% responderam que não possuíam uma união estável, $56,3 \%$ eram casados ou apenas moravam com o parceiro. 0 restante $(15,8 \%)$ não respondeu a essa questão e isso não apresentou relação com os sintomas osteomusculares. No que tange à atividade física, 55,5\% relataram a prática de exercícios como caminhada, ciclismo, musculação e dança. Já os $44,4 \%$ restantes não praticavam nenhum tipo de atividade. A presença de sintomas osteomusculares foi independente da realização de atividade física.

A média de alunos por sala de aula foi de 23,2 \pm 8,3, a média do número de classes por professor foi de $1,9 \pm 1,3$ e o tempo médio de atuação foi de 12,8 $\pm 7,0$ anos de profissão, dos quais apenas o número de classes apresentou relação com os sintomas osteomusculares nos últimos 12 meses $(\mathrm{p}=0,02)$.

Com relação às redes de ensino que lecionavam (municipal, estadual ou particular), 75,3\% dos professores responderam atuar apenas na rede municipal, $18,2 \%$ em duas redes, $0,79 \%$ em três e 5,5\% não responderam essa questão.

Por meio da análise dos resultados obtidos pelo questionário nórdico, observou-se que $91 \%$ dos professores já foram acometidos por sintomas osteomusculares. Destes, mais da metade $(51,5 \%)$ relataram dor na região lombar nos últimos 12 meses, sendo essa a região do corpo mais afetada, seguida por região dorsal e ombros, ambos com 49,2\% dos professores. A região do pescoço foi afetada em 47,6\% dos participantes, os punhos, mãos e dedos em $42 \%$, tornozelos e pés em $41,2 \%$, os joelhos em $33,3 \%$, quadris e coxas em $23,8 \%$, e os cotovelos em $11,9 \%$. Os resultados estão apresentados na Figura 1.

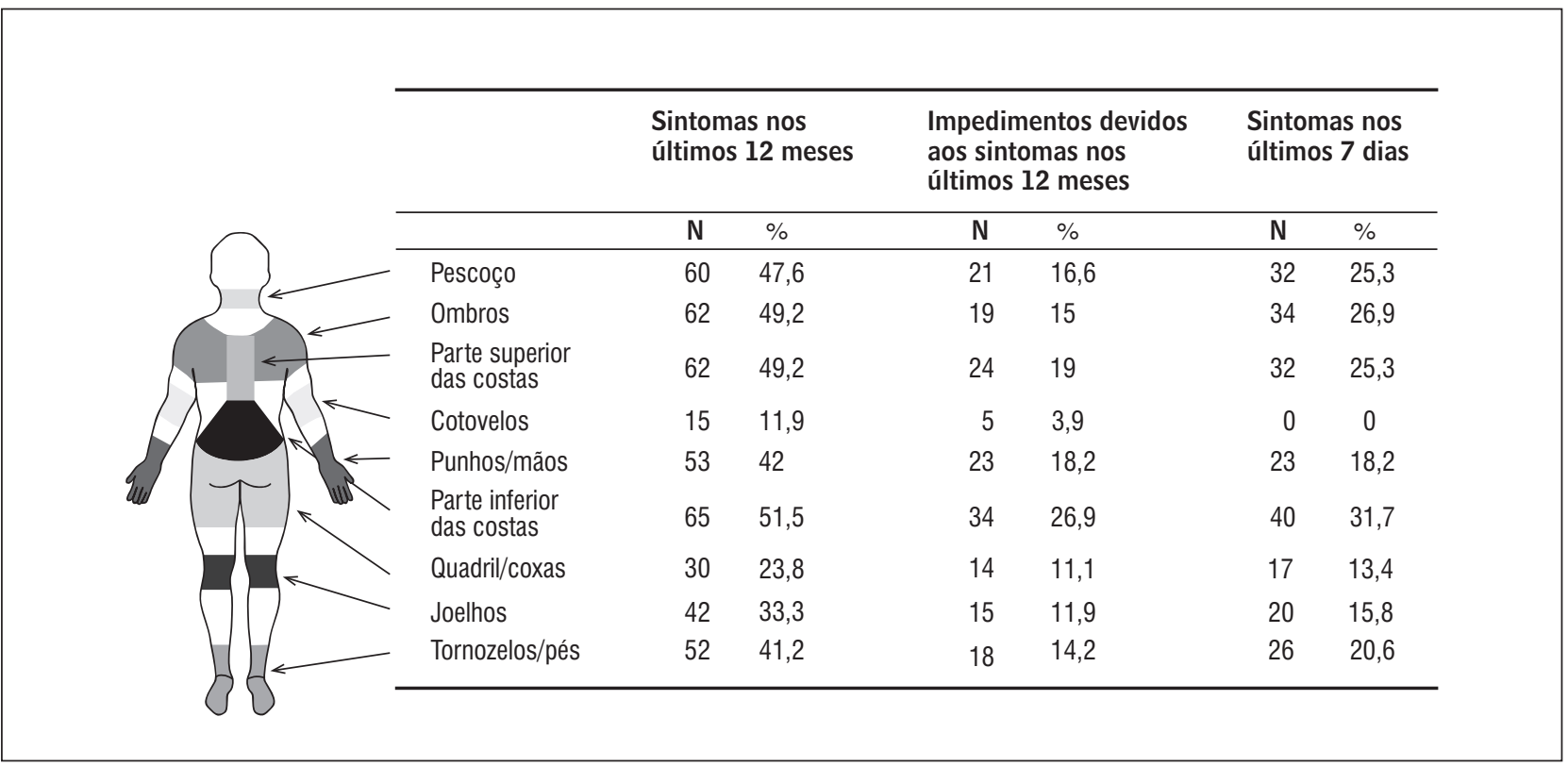

Figura 1 - Sintomas osteomusculares e impedimento para realizar atividades diárias Fonte: Dados da pesquisa. 
Ainda, de acordo com o questionário nórdico, a porcentagem de participantes impedidos de realizar suas atividades domésticas e/ou trabalho e/ou de lazer, nos últimos 12 meses, em virtude de dor na região lombar foi de $26,9 \%$, seguida da região dorsal com $19 \%$, e punhos, mãos e dedos por $18,2 \%$. 0 pescoço foi responsável por $16,6 \%$ dos impedimentos, os ombros por $15 \%$, tornozelos e pés por $14,2 \%$, os joelhos por $11,9 \%$, quadris e coxas por $11,1 \%$, e cotovelo por $3,9 \%$, como pode ser observado na Figura 1.

Nos sete dias precedentes ao questionário, a região lombar também foi a mais afetada, atingindo $31,7 \%$ dos professores, posteriormente os ombros, com $26,9 \%$. 0 pescoço e a região dorsal foram afetados em $25,3 \%$ dos professores, tornozelos e pés em $20,6 \%$, punhos, mãos e dedos em $18,2 \%$, os joelhos em $15,8 \%$, os quadris e coxas em $13,4 \%$ e o cotovelo não teve nenhuma queixa (Figura 1).

Como pode ser observado na Tabela 1 , foram encontradas diferenças significativas $(p<0,05)$ dos sintomas osteomusculares em todas as comparações: impedimento de realizar atividades nos últimos 12 meses com presença de dor nos últimos 12 meses, consulta a algum profissional da saúde nos últimos 12 meses com presença de dor nos últimos 12 meses e consulta a algum profissional da saúde nos últimos 12 meses com problemas nos últimos sete dias, exceto na relação entre a consulta a algum profissional da saúde nos últimos 12 meses com problemas nos últimos sete dias na região do pescoço $(p=0,1624)$.

A variável independente do questionário de dados gerais que teve relação com as variáveis respostas do questionário nórdico, de acordo com a regressão logística, foi o número de classes em que leciona ( $p=0,02)$, não havendo influência significativa de idade, atividade física, número de alunos e o tempo de profissão $(p>0,05)$.

\section{Discussão}

0 presente estudo permitiu identificar os seguintes achados: a região corporal de maior prevalência de sintoma osteomuscular foi a lombar, seguida de região dorsal, ombros e cervical; o número de classes por professor teve relação com a presença de sintomas osteomusculares; os sintomas osteomusculares identificados estão associados ao impedimento de realizar atividades diárias e à assistência por um profissional da saúde.

De acordo com os resultados, pode-se constatar que a maior parte da amostra foi de participantes do gênero feminino. No coletivo de professores de ensino fundamental e médio, as mulheres representam a grande parcela, como pode ser constatado em outros estudos $(1,13,17,18,19)$.

Tabela 1- Relação entre as variáveis do questionário nórdico

\begin{tabular}{|c|c|c|c|}
\hline Regiões do corpo & $\begin{array}{l}\text { Impedimento de realizar } \\
\text { atividades nos últimos } 12 \\
\text { meses/presença de dor nos } \\
\text { últimos } 12 \text { meses }\end{array}$ & $\begin{array}{l}\text { Consulta a algum profissional } \\
\text { da saúde nos últimos } 12 \text { meses/ } \\
\text { presença de dor nos últimos } \\
12 \text { meses }\end{array}$ & $\begin{array}{l}\text { Consulta a algum profissional } \\
\text { da saúde nos últimos } 12 \text { meses/ } \\
\text { problemas nos últimos } 7 \text { dias }\end{array}$ \\
\hline Ombro & $92,67080-5^{\star}$ & $27,53790-5^{\star}$ & $149,7965-5^{\star}$ \\
\hline Pescoço & $5,28237-5^{*}$ & $1828,98150-5^{*}$ & 0,1624 \\
\hline Punhos, mãos, dedos & $0,52873-5^{\star}$ & $25,45610-5^{*}$ & $0,011971-5^{*}$ \\
\hline Região dorsal & $201,73300-5^{\star}$ & $481,38700-5^{\star}$ & $57,925-5^{\star}$ \\
\hline Região lombar & $3,45772-5^{*}$ & $105,74770-5^{*}$ & $168,2722-5^{*}$ \\
\hline Joelhos & $0,85547-5^{*}$ & $0,03235-5^{*}$ & $0,22543-5^{\star}$ \\
\hline Tornozelos e pés & $3,29288-5^{*}$ & $2,56674-5^{\star}$ & $189,3502-5^{\star}$ \\
\hline Cotovelo & $0,91195-5^{*}$ & $7,49493-5^{\star}$ & $0,00000 *$ \\
\hline
\end{tabular}

Legenda: * $=p \leq 0,05$.

Fonte: Dados da pesquisa. 
O tempo médio de atuação na profissão foi de 12,9 anos, aproximado ao que os estudos de Reis et al. (20), Silvany Neto et al. (21) e Cardoso et al. (13) encontraram: 14,4,10,4 e 11 anos, respectivamente. Já Gasparini, Barreto e Assunção (3) observaram uma média um pouco mais alta em Salvador: 16,6 anos de atuação.

A prevalência de distúrbios osteomusculares encontrada nesta investigação foi de 91\%. Carvalho e Alexandre (1), num estudo realizado no interior do estado de São Paulo, apresentaram que de 157 professores do ensino fundamental, 90,4\% relataram sintomas osteomusculares nos últimos 12 meses. Fernandes, Rocha e Costa-Oliveira (12) também encontraram uma alta prevalência, 93\% dos professores do ensino fundamental participantes do estudo eram atingidos por sintomas musculoesqueléticos nos últimos 12 meses, na cidade de Natal (RN). Já a pesquisa realizada por Cardoso et al. (13), com professores do ensino básico da Bahia, identificou uma prevalência menor de acometimentos, $55 \%$ dos sujeitos apresentaram sintomas musculoesqueléticos. Essa variação entre os estudos pode estar relacionada aos materiais e equipamentos disponíveis para os professores utilizarem durante as aulas. Mas essa afirmação apenas pode ser confirmada por meio de uma análise ergonômica dos ambientes de ocupacionais estudados. 0 esforço físico exercido pelos professores também é considerado um fator relevante em relação aos sintomas osteomusculares apresentados, bem como o tempo de profissão e a carga horária semanal (13).

Autores observaram que a dor osteomuscular tem ligação com queda de produtividade, alto índice de absenteísmo, invalidez, modificações nas rotinas de trabalho, lazer ou limitações da vida diária $(4,22,23)$, gerando, ainda, custos econômicos adicionais sobre os sistemas de seguridade social (24).

0 estudo de Porto et al. (8), realizado pela análise de prontuários de professores atendidos pelo Centro de Estudos da Saúde do Trabalhador (CESAT), verificou que o segundo principal diagnóstico das doenças ocupacionais foram os distúrbios osteomusculares (26\% dos sujeitos), principalmente em MMSS. Os mesmos autores afirmaram que os principais riscos associados a esses sintomas foram movimentos repetitivos, postura inadequada e trabalho em pé. A sobrecarga e, por consequência, os sintomas podem ocorrer em virtude da utilização excessiva de grupos musculares específicos em movimentos repetitivos, da manutenção por tempo prolongado de segmentos do corpo em determinadas posições. 0 desconforto e a dor são potencializados quando essas posições exigem esforço ou resistência das estruturas musculoesqueléticas contra a gravidade (25).

Neste estudo, o sintoma mais presente foi a dor na região lombar, em $51,5 \%$ dos participantes. Esse alto índice de dor lombar corrobora com outros estudos (26-29), que afirmam que a dor lombar é uma das mais comuns afecções musculoesqueléticas, e que sua importância pode ser constatada pelas medidas de prevalência e incidência na população geral de adultos e em comunidades de trabalhadores. Fernandes, Rocha e Costa-Oliveira (12) relataram que $53,7 \%$ dos professores apresentaram dor lombar. Cardoso et al. (13) encontraram uma prevalência um pouco menor, $41,1 \%$ dos professores da rede municipal de ensino de Salvador (BA) sofrem com dores nas costas.

As causas do aparecimento dos sintomas osteomusculares podem ser inúmeras; além disso, o presente estudo, por seu caráter transversal e pelo fato de a avaliação ter sido realizada por meio de questionário, não houve diagnóstico etiológico. Entretanto, alguns fatores de risco são comuns para a presença de dor lombar, como trabalho repetitivo, posturas inadequadas, insatisfação no trabalho e esforço físico (26). 0 estudo de Cardoso et al. (13) aponta como fatores que interferem na dor nas costas em professores o tempo de trabalho maior que cinco anos, esforço físico intenso, mobiliário inadequado e calor em sala de aula.

Também verificamos que, apesar de a região lombar ter sido a mais atingida, a maior parte dos acometimentos se deu na parte superior do corpo, atingindo a região dorsal e ombros em $49,2 \%$, pescoço em $47,2 \%$, e punhos, mãos e dedos em $42 \%$ dos participantes. Apenas os cotovelos tiveram um baixo índice de acometimentos, com 11,9\%. Fernandes, Rocha e Costa-Oliveira (12) obtiveram valores um pouco maiores, com $58,7 \%$ atingidos na região dorsal, $50,4 \%$ nos ombros, $53,7 \%$ no pescoço, $51,2 \%$ nos punhos, mãos e dedos, e praticamente o mesmo valor em cotovelos (11,6\%). O número de alunos é apontado por Cardoso et al. (13) como um fator de risco a sintomas osteomusculares em MMSS, sendo que quanto maior o número de alunos, maior o risco. A hipótese para isso é de que o maior número de alunos gera maior esforço por parte dos professores para planejamento e correção de atividades escolares. Queixas com relação ao posicionamento inadequado e incômodo da cabeça e braços foi apontado por 
$53,4 \%$ dos professores participantes $(\mathrm{n}=250)$ do estudo de Delcor et al. (30).

A parte inferior do corpo foi menos acometida pelos sintomas osteomusculares, atingindo quadris e coxas em $23,8 \%$, joelhos em $33,3 \%$ e tornozelos e pés em $41 \%$. As porcentagens encontradas corroboram as do estudo de Fernandes, Rocha e Costa-Oliveira (12), de $25,6 \%$ dos professores com sintomas no quadril e coxas, $38,4 \%$ nos joelhos e $52,9 \%$ nos tornozelos e pés. Cardoso et al. (13) verificaram que $41,1 \%$ dos professores participantes apresentavam sintomas musculoesqueléticos nos membros inferiores (MMII). Já Delcor et al. (30) observaram que $96,7 \%$ dos professores participantes de seu estudo se queixaram por permanecer em pé por longos períodos em sala. Essa queixa pode ser considerada um dos agravantes dos sintomas presentes em MMII.

De acordo com a avaliação realizada com o questionário nórdico, notou-se que todas as regiões do corpo, com exceção dos cotovelos, haviam acometido os professores nos últimos sete dias anteriores à pesquisa. Dos 250 professores que responderam a pesquisa de Delcor et al. (30), 39,4\% mencionaram problemas de saúde nos 15 dias anteriores. Segundo os autores, os professores que apresentam dor nos últimos dias estudados podem representar uma parcela dessa população com alto índice de absenteísmo.

As características laborais dos professores, como o grande esforço físico e os fatores biomecânicos, como atividades repetitivas e realizadas em locais não ergonômicos, podem potencializar os sintomas $(7,13)$.

Ainda foi observado que presença de dor em todas as regiões corporais avaliadas nos últimos 12 meses teve relação com o impedimento de realizar atividades laborais e da vida diária, demonstrando a limitação causada pelo sintoma osteomuscular. Delcor et al. (30) registraram que o percentual de professores com diagnósticos médicos de saúde desde que começaram a trabalhar foi elevado. Batista et al. (19) realizaram um estudo retrospectivo em que analisaram 1.384 fichas médicas individuais de professores da rede municipal de João Pessoa (PB) e encontraram uma média de licenças de 4,6 por professor durante um período de 20 anos. Os principais fatores responsáveis pelas licenças foram os transtornos mentais, com $16,48 \%$, seguido das doenças do sistema osteomuscular e do tecido conjuntivo, com 15,49\%. Em Florianópolis (SC), uma análise realizada em prontuários de professores do ensino fundamental da rede pública afastados das atividades laborais constatou que as duas principais causas de afastamento foram doenças do sistema respiratório e, em seguida, problemas do aparelho locomotor (31). Já em Belo Horizonte, foram registrados 4.279 professores afastados de suas funções laborais, entre o período de maio de 2002 e abril de 2003, sendo que, desses afastamentos, $10,5 \%$ se deram em decorrência de motivos de sintomas osteomusculares, ocupando o terceiro lugar entre os diagnósticos, ressaltando que um mesmo professor pode ter se afastado mais de uma vez (3).

0 estudo de Carvalho e Alexandre (1) afirma que o alto índice de impedimento para a realização das atividades diárias e a procura por algum profissional da área da saúde sugerem que os sintomas osteomusculares representam um risco ocupacional para os professores.

Foi encontrada relação significativa entre a presença de dor nos últimos 12 meses e nos últimos sete dias com a consulta a algum profissional da saúde. Isso pode significar que a dor é persistente, prejudica a qualidade de vida das professoras e onera o sistema público de saúde.

Cardoso et al. (13) não constataram relação significativa entre o número de salas que os professores lecionam e os sintomas osteomusculares. Contudo, no presente estudo o número de classes apresentou relação com o impedimento de realizar atividades profissionais e da vida diária em razão dos sintomas, fato que pode ser explicado pelo desgaste biopsicossocial dos professores, causando algum sintoma osteomuscular (3).

As questões verificadas por meio do questionário de dados gerais (idade, estado civil, atividade física, número de alunos por sala, rede de ensino e tempo de profissão) não apresentaram diferença significativa com os sintomas osteomusculares nos últimos 12 meses, impedimento para realização das atividades normais, consulta a algum profissional da saúde e presença de dor nos últimos sete dias. Houve a exceção apenas do número de classes, que indicou relação com os sintomas nos últimos 12 meses.

Outro estudo encontrou resultados diferentes, apresentando relação entre a idade e os sintomas osteomusculares. Professores com idade acima de 40 anos tiveram maior prevalência de dor na investigação feita na Bahia (13). 0 mesmo estudo justificou que esse achado pode ter se dado em virtude do processo de envelhecimento do sistema osteomioarticular. Reis et al. (11) obtiveram ligação significativa entre 
idade $\geq 27$ anos e cansaço mental e nervosismo - de acordo com Melzer (9), existe essa associação entre fatores psicossociais e distúrbios osteomusculares relacionados ao trabalho.

No que se refere à prática de atividade física, verificou-se que 55,5\% praticavam exercícios como caminhada, ciclismo, musculação e dança, e os $44,4 \%$ restantes não praticavam nenhum tipo de atividade. Carvalho e Alexandre (1) corroboram com os achados com porcentagens próximas, sendo $53,5 \%$ praticantes de exercícios físicos e $46,5 \%$ não praticantes. Os autores afirmaram que as justificativas para a não realização de exercícios podem ser falta de tempo, dupla jornada de trabalho ou questões socioeconômicas. 0 mesmo estudo também não encontrou relação significativa entre a prática de atividade física e a presença de sintomas. Já o estudo de Fernandes, Rocha e Costa-Oliveira (12) descreveu relação significativa entre a presença de sintomas osteomusculares nos últimos 12 meses e a prática inadequada de atividade física. Com isso, surge a hipótese de que os sujeitos participantes dos estudos apresentam hábitos de atividade física diferentes no que se refere a intensidade, tipo de atividade, volume e frequência.

Houve relação significativa entre o impedimento de realizar atividades normais e a procura por algum profissional da saúde. A incapacidade para trabalhar faz com que as pessoas busquem tratamento, onerando o sistema de saúde público e privado (32).

De acordo com o relato dos professores a respeito das redes de ensino em que lecionavam (municipal, estadual ou particular), 75,3\% dos professores afirmaram atuar em apenas uma rede, 18,2\% em duas redes, 0,79\% em três. Carvalho e Alexandre (1) encontraram que 19\% trabalhavam em duas ou três redes, valor muito próximo do verificado no presente estudo. Esses autores ainda afirmam que baixa remuneração e instabilidade podem ser motivos que levam os professores a lecionar em várias escolas. Está de acordo com essa afirmação Delcor et al. (30), que constatou um percentual de $26,2 \%$ dos professores trabalhando em mais de uma escola privada e $28,4 \%$ trabalhando em outras escolas da rede estadual ou municipal, além da privada, para aumentar a renda familiar.

Ao contrário do presente estudo, diversos autores identificaram o tempo de profissão como preditor de distúrbios osteomusculares. Cardoso et al. (13) verificaram que o tempo de trabalho maior que 5 anos foi significante na identificação dos sintomas osteomusculares em membros superiores e costas, não sendo significativo para MMII. Carvalho e Alexandre (1) identificaram o tempo de profissão inferior ou igual a 15 anos como preditor. Reis et al. (11) acrescentaram que lecionar há cinco ou mais anos se associou a um maior índice de cansaço mental e nervosismo.

Considerando-se a origem multifatorial da LER/ DORT, o reconhecimento dos fatores de risco presentes na atividade laboral e sua inclusão nas estratégias de tratamento e controle dessas síndromes devem ser mais bem valorizados para se aumentar a resolutividade. Além disso, é importante a uniformização das condutas avaliativas da LER/DORT para fornecer melhor suporte na formação dos profissionais da saúde para maior clareza e segurança diagnóstica desses acometimentos (33).

Assim, os resultados deste estudo podem ser utilizados como parâmetros para o direcionamento de políticas públicas que tenham como objetivo a melhora da saúde do professor.

\section{Conclusão}

Os professores entrevistados para esta pesquisa apresentaram elevada prevalência de sintomas osteomusculares, especialmente nas seguintes regiões: lombar, ombros e região dorsal, tanto em longo como curto prazo. Além disso, essas regiões mais afetadas apresentaram relação com o maior número de afastamentos, número de classes que lecionavam e a assistência por um profissional da saúde.

Os resultados são relevantes para os próprios professores, alertando e orientando o debate sobre as DORT nessa categoria e a discussão de possíveis medidas de prevenção, tratamento e políticas públicas.

\section{Referências}

1. Carvalho, AJFP, Alexandre NMC. Sintomas osteomusculares em professores do ensino fundamental. Rev Bras Fisioter. 2006;10(1):35-41. doi:10.1590/ S1413-35552006000100005.

2. Picoloto D, Silveira E. Prevalência de sintomas osteomusculares e fatores associados em trabalhadores de uma indústria metalúrgica de Canoas-RS. Cienc Saude Colet. 2008;13(2):507-16. doi:10.1590/ S1413-81232008000200026. 
3. Gasparini SM, Barreto SM, Assunção AA. O professor, as condições de trabalho e os efeitos sobre sua saúde. Educ Pesq. 2005;31(2):189-99. doi:10.1590/ S1517-97022005000200003.

4. Maciel, ACC, Fernandes MB, Medeiros LS. Prevalência e fatores associados à sintomatologia dolorosa entre profissionais da indústria têxtil. Rev Bras Epidemiol. 2006;9(1):94-102. doi:10.1590/S1415790X2006000100012.

5. Mendonça HP Jr, Assunção AA. Associação entre distúrbios do ombro e trabalho: breve revisão de literatura. Rev Bras Epidemiol. 2005;8(2):167-76. doi:10.1590/S1415-790X2005000200009.

6. Walsh IAP, Corral S, Franco RN, Canetti EE, Alem MER, Coury HJ. Capacidade para o trabalho em indivíduos com lesões músculo-esqueléticas crônicas. Rev Saúde Pública. 2004;38(2):149-56. doi:10.1590/ S0034-89102004000200001.

7. Souza KR, Santos MBM, Pina JA, Maria ABV, Carmo MAT, Jensen M. A trajetória do Sindicato Estadual dos Profissionais da Educação do Rio de Janeiro (Sepe-RJ) na luta pela saúde no trabalho. Cienc Saúde Colet. 2003;8(4):1057-68. doi:10.1590/ S1413-81232003000400027.

8. Porto LA, Reis IC, Andrade JM, Nascimento CR, Carvalho FM. Doenças ocupacionais em professores atendidos pelo Centro de Estudos da Saúde do Trabalhador (CESAT). Rev Baiana Saúde Pública. 2004;28(1):33-49.

9. Melzer ACS. Fatores de risco físicos e organizacionais associados a distúrbios osteomusculares relacionados ao trabalho na indústria têxtil. Fisioter Pesq. 2008;15(1):24-32.

10. Deliberato PCP. Fisioterapia preventiva: fundamentos e aplicações. Barueri: Manole; 2002.

11. Reis EJFB, Araújo TM, Carvalho FM, Barbalho L, Silva MO. Docência e exaustão emocional. Educ Soc. 2006;27(94):229-53. doi:10.1590/S010173302006000100011 .

12. Fernandes $M H$, Rocha VM, Costa-Oliveira AGR. Fatores associados às prevalência de sintomas osteomusculares em professores. Rev Salud Publica. 2009;11(2):25667. doi:10.1590/S0124-00642009000200010.
13. Cardoso JP, Ribeiro IQB, Araújo TM, Carvalho FM, Reis EJFB. Prevalence of musculoskeletal pain among teachers. Rev Bras Epidemiol. 2009;12(4):1-10.

14. Lacaz, FAC. Qualidade de vida no trabalho e saúde/ doença. Cienc Saúde Colet. 2000;5(1):151-61. doi:10.1590/S1413-81232000000100013.

15. Barros ENC, Alexandre NMC. Cross-cultural adaptation of the Nordic musculoskeletal questionnaire. Int Nurs Rev. 2003;50:101-108. doi:10.1046/ j.1466-7657.2003.00188.x.

16. Pinheiro FA, Tróccoli BT, Carvalho CV. Validação do Questionário Nórdico de sintomas osteomusculares como medida de morbidade. Rev Saúde Pública. 2002;36(3):307-12. doi:10.1590/ S0034-89102002000300008.

17. Ristum M, Bastos ACS. Violência urbana: uma análise dos conceitos de professores do ensino fundamental. Cienc Saúde Colet. 2004;9(1):225-39. doi:10.1590/ S1413-81232004000100022.

18. Werle FOC. Práticas de gestão e feminização do magistério. Cad. Pesq. 2005;(35)126:609-34. doi:10.1590/ S0100-15742005000300005.

19. Batista JBV, Carlotto MS, Coutinho AS, Nobre Neto FD, Augusto LGS. Saúde do professor do ensino fundamental: uma análise de gênero. Cad Saúde Colet. 2009;17(3):657-74.

20. Reis EJFB, Carvalho FM, Araújo EM, Porto LA, Silvany Neto AM. Trabalho e distúrbios psíquicos em professores da rede municipal de Vitória da Conquista, Bahia, Brasil. Cad Saúde Pública. 2005;21(5):1480-90. doi:10.1590/S0102-311X2005000500021.

21. Silvany Neto MAS, Araújo TM, Dutra FRD, Azi GR, Alves RL. Condições de trabalho e saúde de professores da rede particular de ensino de Salvador, Bahia. Rev Baiana Saúde Pública. 2000;24(1-2):45-56.

22. Brandão AG, Horta BL, Tomasi E. Sintomas de distúrbios osteomusculares em bancários de Pelotas e região: prevalência e fatores associados. Rev Bras Epidemiol. 2005;8(3):295-305. doi:10.1590/ S1415-790X2005000300011. 
23. Santos Filho S, Barreto S. Atividade ocupacional e prevalência de dor osteomuscular em cirurgiões dentistas de Belo Horizonte, Minas Gerais, Brasil: contribuição ao debate sobre os distúrbios osteomusculares relacionados ao trabalho. Cad Saúde Pública. 2001;7(1):18193. doi:10.1590/S0102-311X2001000100019.

24. Matos MG, Hennington EA, Hoefel AL, Dias-da-Costa JS. Dor lombar em usuários de um plano de saúde: prevalência e fatores associados. Cad Saúde Pública. 2008;24(9):211522. doi:10.1590/S0102-311X2008000900017.

25. Brasil. Ministério da Previdência Social. [citado 8 Dez. 2010]. Disponível em: http://www.dataprev.gov.br/.

26. Radovanovic CAT, Alexandre NMC. Desenvolvimento de um instrumento para avaliar a movimentação e transferência de clientes: um enfoque ergonômico. Rev Esc Enferm USP. 2002;36(3):231-9. doi:10.1590/ S0080-62342002000300004.

27. Gurgueira GP, Alexandre NMC, Corrêa HR Filho. Prevalência de sintomas músculo-esqueléticos em trabalhadoras de enfermagem. Rev LatinoAm Enfermagem. 2003;11(5):608-13.doi:10.1590/ S0104-11692003000500007.

28. Nordin M, Alexandre NMC, Campello, M. Measures for low back pain: a proposal for clinical use. Rev LatinoAm Enfermagem. 2003;11(2):152-5. doi:10.1590/ S0104-11692003000200002.

29. Abreu AV, Mello AP, Trovão GS, Fontenelle CRC. Avaliação clínico-radiográfica da mobilidade da lordose lombar. Rev Bras Ortop. 2007;42(10):313-23. doi:10.1590/S0102-36162007001000001.
30. Delcor NS, Araújo TM, Reis EJFB, Porto LA, Carvalho FM, Silva MO, et al. Condições de trabalho e saúde dos professores da rede particular de ensino de Vitória da Conquista, Bahia, Brasil. Cad Saúde Pública. 2004;20(1):187-96. doi:10.1590/S0102-311X2004000100035.

31. Siqueira MJT, Ferreira ES. Saúde das professoras nas séries iniciais; o que o gênero tem a ver com isso? Psicol Ciênc Prof. 2003;23(3):76-83. doi:10.1590/ S1414-98932003000300011.

32. Brasil. Ministério da Saúde. Secretaria de Políticas de Saúde. Lesões por esforços repetitivos (LER) Distúrbios osteomusculares relacionados ao trabalho (DORT). Brasília: Ministério da Saúde; 2001.

33. Alencar JF, Coury HJCG, Oishi J. Aspectos relevantes no diagnóstico de DORT e fibromialgia. Rev Bras Fisioter. 2009;13(1):52-8. doi:10.1590/ S1413-35552009005000001.

Recebido: 23/04/2011

Received: 04/23/2011

Aprovado: 09/11/2011

Approved: 11/09/2011 